\title{
L'imaginaire de la corruption dans La Pratique de Repentance (1631) de Nicolas Vignier
}

\author{
Inès Kirschleger
}

\section{(2) OpenEdition \\ 1 Journals}

Édition électronique

URL : http://journals.openedition.org/edl/915

DOI : $10.4000 /$ edl. 915

ISSN : 2296-5084

Éditeur

Université de Lausanne

\section{Édition imprimée}

Date de publication : 15 décembre 2015

ISBN : 978-2-940331-47-5

ISSN : 0014-2026

\section{Référence électronique}

Inès Kirschleger, "L'imaginaire de la corruption dans La Pratique de Repentance (1631) de Nicolas

Vignier », Études de lettres [En ligne], 3-4 | 2015, mis en ligne le 01 décembre 2018, consulté le 15

décembre 2020. URL : http://journals.openedition.org/edl/915; DOI : https://doi.org/10.4000/edl.915

\section{(c) Études de lettres}




\section{L'IMAGINAIRE DE LA CORRUPTION \\ DANS LA PRATIQUE DE REPENTANCE (1631) DE NICOLAS VIGNIER}

En 1631, le pasteur de l'Eglise Réformée de Blois Nicolas Vignier publie un ensemble de vingt sermons sur le psaume 51 sous le titre de Pratique de Repentance. Le recueil se présente, dans la tradition des commentaires exégétiques fin de siècle, comme une succession de méditations sur chacun des versets du psaume. L'objectif de l'auteur est double: joindre l'instruction à l'utilité, ou en d'autres mots le discours exhortatif du prédicateur ("amendez-vous») à la réflexion du théologien sur l'«excellence» du livre des psaumes. Vignier propose ainsi, selon les termes de son Epître liminaire, de "contempler un parfait tableau de la vraye repentance du péché» au prisme du "plus clair miroir de la grace \& misericorde de Dieu» que les Ecritures puissent proposer. Ce faisant les méditations de Vignier placent le concept de corruption "generale" et "universelle» de la nature humaine au cœur de la problématique de la repentance. Tout un réseau de convergences imaginaires puisées dans les mots et dans l'esprit des psaumes affleure ainsi autour de la notion de corruption, sur cette scène dramatique où se joue la repentance du pécheur pénitent.

Si le livre des psaumes constitue un excellent support pour méditer la représentation du péché et s'interroger sur les conséquences d'une âme dépravée dans la pratique de la foi, à plus forte raison le psaume 51, grand psaume de la pénitence s'il en fut ${ }^{1}$, psaume du déchirement de l'âme repentante rongée par la culpabilité et le remords, se prête-t-il particulièrement bien à une analyse du traitement de la notion de corruption dans la prédication réformée du premier XVII e siècle. Par la place qu'il occupe dans la liturgie et la tradition chrétiennes, le Miserere

I. Psaume essentiellement connu chez les réformés sous l'incipit «Misericorde au paouvre vicieux", dans la traduction de Clément Marot. 
mei est assurément l'un des psaumes les plus connus et les plus prisés du genre de la méditation. Les protestants semblent toutefois l'avoir moins plébiscité que les catholiques, l'attention portée aux psaumes dits pénitentiels d'une façon globale étant chez eux bien moindre que chez leurs coreligionnaires. Mais si l'on ne peut pas dire qu'il s'agisse là d'un psaume de prédilection, le psaume 51 figure cependant en bonne part chez les réformés, nourrissant bon nombre de méditations à la fin du $\mathrm{XVI}^{\mathrm{e}}$ siècle $^{2}$, puis essentiellement des sermons au siècle suivant ${ }^{3}$. La prédication réformée, dans ses titres et dans ses citations, retiendra de ce psaume essentiellement des images (le cœur contrit et froissé, l'hysope, l'eau de la grâce) ${ }^{4}$, des gestes (le lavement, l'ouverture des lèvres) ${ }^{5}$, des couleurs (le rouge sang, la blancheur de la neige, le noir péché) ${ }^{6}$, ainsi que les notions de sacrifice et de repentance ${ }^{7}$. Le recueil publié en 1631

2. Voir Th. de Bèze, Meditation sur le LI Psalme; Ph. Duplessis-Mornay, Meditation sur le Pseaume LI; A. d'Aubigné, Meditation sur le Pseaume LI; ainsi que V. Ferrer, «Variations autour du Psaume 51 ».

3. Citons par ordre chronologique (abrégés en Serm. Ps 51, suivi du numéro des versets) : Serm. Ps 51, 12-17 par Jean Daillé, prononcé à Charenton en janvier 1641 (Mélange de sermons); Serm. Ps 51, 17 par Jérémie Viguier (L'ouverture des levres ou Sermon sur le Pseaume 51. vers. 17. Par Jeremie Viguier Ministre de la Parole de Dieu en l'Eglise Réformée de Nerac); Serm. Ps 51, 1-2 par Pierre Mussard, prononcé en août 1672 (Sermons sur divers textes de la Saincte Escriture); Serm. Ps 51, 19 par François Turrettin, sermon de préparation d'un jeûne (Sermons sur divers passages de l'Escriture Sainte); Serm. Ps 51, 3 par Alexandre Morus (publication posthume, Sermons choisis de Monsieur Morus sur divers textes de l'Ecriture sainte); Serm. Ps 51, 9 par Pierre Du Bosc (Sermons sur divers textes de l'Ecriture sainte convenables au temps).

4. Voir dans la traduction en prose de la Bible de Genève de 1588 (qu'utilise Vignier dans son recueil), les versets suivants: verset 19: "ô Dieu, tu ne mesprises point le cœur froissé $\&$ brisé»; verset 9 , "purge-moy de péché avec hyssope, $\&$ je serai net». Voir aussi dans la traduction de Marot, strophe $1:$ «me nettoyer d'eau de grace te plaise».

5. Verset 4: «Lave-moi tant \& plus de mon iniquité, \& me nettoye de mon péché»; verset 17, "Seigneur, ouvre mes levres, $\&$ ma bouche annoncera ta loüange».

6. Verset 16: «delivre-moi de tant de sang»; verset 9, «lave-moi, \& je serai plus blanc que neige». Voir aussi dans la traduction de Marot, strophe 2, «mon péché se présente incessamment noir \& laid devant moy", et strophe 4, "tu laveras ma trop noire macule».

7. Verset 19: «les sacrifices de Dieu sont l'esprit froissé»; verset $21:$ «tu prendras plaisir aux sacrifices justement faits». Et dans la traduction de Marot, strophe 9: «Le sacrifice agreable \& bien pris de L'Eternel, c'est une ame dolente, un cœur submis, une ame penitente». Les citations de psaumes seront toutes extraites de la version en vers 
par le pasteur Nicolas Vignier sous le titre de Pratique de repentance ${ }^{8}$, regroupant un ensemble de vingt sermons successifs sur le psaume 51, verset après verset, nous a paru dans ce contexte particulièrement digne d'intérêt, et ce pour deux raisons au moins: d'une part, Vignier est à notre connaissance le seul prédicateur réformé à avoir publié au XVII siècle un si grand nombre de sermons sur un même psaume; d'autre part, son recueil illustre admirablement la pratique de la lectio continua, laquelle consiste à développer en série, au fil du texte, une suite de sermons sur un même livre biblique (le cas de Vignier est, pour le XVII ${ }^{\mathrm{e}}$ siècle, l'un des rares exemples qui nous soit parvenu sous cette forme). $\mathrm{La}$ Pratique de Repentance a sans doute rencontré un certain succès puisque

de Clément Marot et Théodore de Bèze, en raison de son évidente charge poétique par rapport à la version en prose, ce qui permet de mieux repérer certaines constantes dans l'ordre de l'imaginaire (Cl. Marot, Th. de Bèze, Les Psaumes en vers français avec leurs mélodies).

8. Voir l'article "Vignier» in E. et E. Haag, La France Protestante ou vie des protestants français qui se sont fait un nom dans l'Histoire, t. IX, p. 493-496. L'article mentionne trois générations de Nicolas Vignier: le premier Nicolas (1530-1596) exerça la médecine en Allemagne où il s'était réfugié après sa conversion au protestantisme, avant de rentrer en France et de devenir médecin d'Henri III, en même temps qu'historiographe de France et conseiller d'Etat. Les deux Nicolas suivants (fils et petit-fils du précédent) furent tous deux pasteurs de l'Eglise Réformée de Blois. Celui qui nous intéresse, Nicolas père, desservit vraisemblablement cette paroisse dès 1601 et toute sa vie durant. Il institua son fils pasteur dans cette même paroisse, mais ce dernier mourut à l'âge de vingt-quatre ans. On ne sait que peu de choses sur Nicolas père, si ce n'est qu'il présida plusieurs synodes $(1611,1612,1618,1638)$ et qu'il a laissé l'image d'un "ennemi ardent, violent même, de l'Eglise romaine» (ibid, p.494). Il reste de lui, outre un Theatre de l'Antechrist, auquel est répondu au cardinal Bellarmin, essentiellement des sermons: la Pratique de repentance, mais aussi un recueil intitulé Le Pescheur d'hommes, ou Du devoir \& des qualitez des Ministres de la Parole de Dieu. Avec le formulaire de la Reception \& Confirmation des Pasteurs, selon la Discipline des Eglises Reformées de ce Royaume, et une décade de sermons de préparation à la Cène. Nous utilisons l'édition de 1671, La Pratique de Repentance, ou Sermons sur le Pseaume 51, avec une paraphrase d'iceluy. Par Nicolas Vignier. ITEM La Recherche du Cour, ou cinq Sermons sur le 10 verset du 17. chapitre de Jeremie. Le Bon Centenier, ou cinq sermons sur sept versets du chap. 8 de S. Mathieu. Par Nicolas Vignier son Fils, tous deux Ministres du S. Evangile en l'Eglise Reformée de Bloys. Se vend à Charenton, Par Estienne Lucas. L'ouvrage a été publié en 1631 à La Rochelle, puis réédité à Rouen en 1650. Nous avons également recensé une édition de 1670, "Se vend Par Thomas Le Gentil, demeurant à Paris ruë des Noyers, \& à Charenton en la boutique de Daniel du Chemin, qui est la premiere en sortant du Temple à main droite» (Bibliothèque de la Société de l'Histoire du Protestantisme Français, cote $\left.8^{\circ} 1384\right)$. 
nous en connaissons au moins trois rééditions (1650, 1670 et 1671). Ces sermons ne semblent pas avoir été prononcés à l'occasion de célébrations particulières; seul le dixième sermon fait allusion au sacrement de la Cène, mais le recueil n'offre aucune indication complémentaire de date ou de circonstance à ce sujet ${ }^{9}$. Comme l'indique le titre du recueil, la visée du prédicateur est essentiellement pratique, car le pire ennemi de la foi est pour lors en nous-mêmes, dans nos cœurs «soüillez de lasciveté, pressez d'envie, rongez d'avarice, brûlans d'ambition, enflez d'orgueil, bouffis de vanité, crevans de haine $\&$ d'animosité contre nos freres " ${ }^{10}$. Satan rôde et veille, mais ne saurait pourtant porter atteinte aux "eleus de Dieu ": «le Ciel ne le veut pas; l'Enfer ne le peut " ${ }^{11}$. Il est donc de la plus haute nécessité de se convertir, de laisser mourir "nôtre nature corrompuë», et de passer enfin "de la puissance de Satan à Dieu» "2. Mais si le relâchement des mours et la dépravation des âmes sont des thématiques privilégiées de la littérature sermonnaire, la réflexion morale, chez Vignier, s'élabore à partir de tout un imaginaire dynamique de la corruption qui vise à enrayer le processus naturel et constant par lequel, selon lui, l'âme humaine se plaît et se complaît dans le péché. Et si le concept de corruption revêt une acception commune et générale, liée à la doctrine du péché originel, toute une constellation d'images puissantes affleure dans le même temps sur cette scène dramatique où se joue la repentance de l'homme pécheur. C'est ce réseau que nous souhaiterions suivre pas à pas: après avoir dégagé les enjeux de l'écriture de Vignier à partir du choix du livre des psaumes, nous verrons comment ces psaumes précisément assurent la cohérence imaginaire et l'efficacité rhétorique du discours.

9. Serm. X, p. 167: «Dieu crie des Cieux de nôtre Seigneur Jesus-Christ, Cetuy-ci est mon Fils bien aimé [...]. Sa passion est nôtre satisfaction, sa mort nôtre rédemption, le Sacrement de l'Eucharistie auquel nous avons ce jourd'huy participé le memorial de sa mort, dont la celebration nous fournit par conséquent les mêmes usages que fait la meditation de la mort de Christ».

Io. Serm. X, p. 167.

II. Ibid.

I2. Ibid., p. 166. 
Enseigner par l'exemple de David: l'excellence et l'utilité du livre des psaumes

La visée didactique qui sous-tend l'écriture de La Pratique de repentance s'affiche d'emblée dans l'épître liminaire: «instruction", «enseignement ", "utilitée sont les maîtres-mots de cet écrit préfaciel qui annonce des sermons destinés à être "de singuliere utilité à l'Eglise», et "de plus grand usage» pour chaque fidèle dans sa vie quotidienne ${ }^{13}$. Ce qui a été prodigué "de bouche" pourra ainsi, par l'acte de publication qui permettra une lecture renouvelée autant de fois que nécessaire, être "pesé», "médité» et "serré" dans le cœur de chacun. L'épître contient également l'essentiel du cadre théologique qui détermine la réflexion générale de l'ouvrage: depuis qu' «Adam est tombé en la mort par le péché», sa "miserable postérité» est inévitablement portée vers le "mal de péché»; lui-même entraîne à son tour "ce mal de peine qui suit le péché» et qui est cause de douleurs incessantes que le temps et le remords rendent cuisantes si la "pratique de repentance» ne les chasse. Vignier conçoit ainsi ses sermons comme «une Escole» du Ciel, où les fidèles zélés et appliqués pourront «apprendre le chemin " qu'il faut suivre afin de se libérer de la "coulpe» et de la "peine» qui y est si étroitement attachée. Seulement les préceptes qu'il prodigue constituent déjà un niveau avancé de réflexion théologique, non «la premiere leçon du service de Dieu» qui consiste en l'apprentissage de «l'Innocence» ou "Intégrité », mais en quelque sorte une leçon de rattrapage centrée sur les concepts d'«Humilité \& Repentance» ${ }^{14}$.

Les objectifs du discours étant posés, il ne reste plus au prédicateur qu'à justifier le choix d'un psaume comme support de l'entreprise sermonnaire, ce qui s'obtient aisément si l'on considère d'une part la figure de David, "grand Roy", "grand Prophete, homme selon le cœur de Dieu, le cœur, la langue $\&$ la plume du Roy des Rois» ${ }^{15}$, «si grand saint pecheur» et "si grand pecheur repentant» ${ }^{16}$, et d'autre part la nature du livre des psaumes, cet abrégé de toutes les richesses des Ecritures, qui

I3. Epître dédicatoire datée du premier janvier 1631: «A tous les Fideles de l'Eglise Reformée de Blois, Mes tres-chers \& bien-aimez Freres, grace \& paix de par Dieu nôtre pere, \& de par nôtre Seigneur Jesus-Christ ", [n. p.]

I4. Ibid.

I5. Ibid.

I6. Serm. I, p. 13. 
jouit depuis les Enarrationes in Psalmos de saint Augustin d'un très haut prestige. Le premier sermon de Vignier revendique d'ailleurs clairement cette filiation en proposant plusieurs citations d'Augustin, comme l'indiquent les marges du texte. Ces extraits ont pour but de poursuivre la présentation du livre des psaumes que l'épître avait amorcée:

Désirez-vous voir une magnanimité héroïque, une Justice exquise, une grave moderation, une exacte prudence, une patience invincible? Voulez-vous apprendre les mysteres de Dieu, les souffrances de Christ, les terreurs de l'ire, les consolations de la grace, les œuvres de la Providence sur le monde, les joyes du siecle à venir? Mais surtout voulez-vous contempler un parfait tableau de la vraye repentance du péché? Vous trouverez tout cela és Pseaumes de David; et ce dernier cy par excellence en ce Pseaume cinquante $\&$ unième ${ }^{17}$.

et d'asseoir en même temps le discours du prédicateur sur une caution sûre que nul ne songerait à lui contester:

[...] le livre des Pseaumes contient en soy tout ce qui se peut tirer d'utilité de tous les autres. [August. Prologo in Psal.] Il predit les choses à venir, il raconte les gestes des anciens, il donne loy aux vivans, il ordonne ce qui est à faire, c'est un tresor commun de bonne doctrine, qui administre proprement ce qui est necessaire à chacun. [...] Le Pseaume est la tranquillité des ames, un porte enseigne de paix, qui arreste les troubles \& les flots de nos pensées, [...]. Il chasse les Demons, il invite les Anges à notre aide. C'est un bouclier és terreurs de la nuit, un repos aux travaux du jour. [...]. C'est la voix de toute l'Eglise, l'occupation des Anges, le parfum spirituel des armees celestes [...]. Bref je vous ose dire que l'usage du Soleil, de l'air \& du feu n'est point plus utile à l'entretenement de la vie naturelle, qu'est l'usage des Pseaumes à ceux qui sont amateurs de la vie spirituelle ${ }^{18}$.

Le discours moral, dont la composante injonctive est tirée de l'Evangile de Luc ("Amandez-vous \& croyez à l'Evangile» ${ }^{19}$ ), promeut également l'excellence et l'utilité du livre des psaumes, le plus "parfait tableau de la vraye repentance du péché» et le "plus clair miroir de la grace

17. "Epître dédicatoire», ibid.

I8. Serm. I, p. 2 sq.

19. Luc 24, 47, «Epître dédicatoire», ibid. 
$\&$ misericorde de Dieu" que les Ecritures puissent proposer ${ }^{20}$. Le prédicateur se pose ainsi en serviteur zélé de la Parole de Dieu dont le rôle est de guider ses frères sur la voie qui plaît à Dieu, ce qu'un contemporain de Vignier, Fabrice de la Bassecour, pasteur de l'Eglise française d'Amsterdam, expliquait ainsi dans une adresse aux «Bourgmaistres et Eschevins de la Ville d'Amsterdam»:

Vous estes, Messieurs, des Moyses en l'Eglise de Dieu: \& nous Pasteurs, des Aarons: tous deux serviteurs de Dieu: les uns pour commander la pieté, les autres pour l'enseigner ${ }^{21}$.

L'incipit du premier sermon de Vignier confirme cette perspective que l'épître annonçait:

Il n'y a moyen quelconque d'enseigner les hommes, dont Dieu ne se serve és saintes Ecritures. Il nous instruit par preceptes, il nous alleche par promesses, il nous epouvante par menaces ${ }^{22}$.

"Instruire», "allécher» et "épouvanter» tout à la fois, c'est effectivement ce que Vignier va s'efforcer de faire, afin que chacun prenne conscience de la nécessité impérieuse de se repentir et de «s'amender ». Et c'est dans la conjonction de cette perspective didactique et du choix de l'outil jugé le plus approprié (le livre des psaumes) que réside tout l'enjeu de l'écriture sermonnaire. David servira d'exemple au fidèle, car l'Ecriture, dans sa simplicité brute et touchante, regorge de modèles auxquels le fidèle est appelé bien évidemment à se conformer. Les "cris", "soupirs" et «larmes» du David pénitent "jusques à ce qu'il ait esté assuré de son pardon " ${ }^{23}$ doivent donc édifier le fidèle et lui permettre d'agir en conséquence "à l'avancement de [son] salut» ${ }^{24}$ :

Ainsi tirons-nous des remedes des playes des autres, \& des morts mêmes faisons une mommie spirituelle pour les vivans ${ }^{25}$.

20. Ibid.

2I. Sermons de piété, pour réveiller l'ame à son salut. Par Fabrice de la Bassecour, Ministre en l'Eglise Françoise recueillie à Amsterdam. A Amsterdam, chés Louys Elzevier, "Epître dédicatoire», [n. p.].

22. Serm. I, p. 1.

23. Serm. I, p. 6.

24. "Epître dédicatoire», ibid.

25. Serm. I, p. 6. 
L'incongruité et la violence de la métaphore donnent le ton, en même temps qu'elles frappent l'esprit et imprègnent la mémoire de l'auditeur; ainsi s'explique dans les premiers sermons la récurrence d'images aussi étonnantes que spectaculaires pour dénoncer d'une part la laideur du péché et, d'autre part, les accusations de la conscience qui font naître "horreurs» et "terreurs étranges en l'ame $[\mathrm{du}]$ pecheur» ${ }^{26}$, une fois sorti de la "letargie spirituelle» ${ }^{27}$ dans laquelle le péché tend à le retenir. Certes, en bon théologien réformé, Vignier ne se contente pas de se référer seulement aux versets du psaume ${ }^{28}$, mais sa prose est un véritable conglomérat de personnages, de références et de citations bibliques qui se bousculent en nombre dans le texte et dans ses marges.

\section{Au fond du "gouffre de désespoir»"29}

Dès le premier sermon en effet, puisant à diverses sources vétérotestamentaires, Vignier joue des effets d'accumulation et de gradation que favorisent des images grandioses, comme peuvent l'être l'infini de l'océan ou le songe de Nabuchodonosor (Daniel 2, 31-33):

Ainsi les pechez s'entresuivent l'un l'autre, comme les vagues de la mer s'entrepoussent l'une l'autre, le sang touche le sang, le meurtre touche le meurtre: comme parloit le Prophete Osée chap. 4. Et quand un pécheur s'est une fois abandonné à ses corruptions \& aux tentations de Satan, il devient semblable à cette image que vid Nebucadnetsar en songe, qui avoit la teste d'or, la poitrine d'argent, le ventre d'airain, les jambes de fer, les pieds de terre; c'est à dire, il va toûjours de pis en pis, jusqu'à ce que la pierre coupée sans main de la montagne, c'est à dire, le jugement immediat de Dieu, le mette en pieces, comme un vaisseau de terre, $s^{\prime} i l$ ne le previent par sa repentance ${ }^{30}$.

Du «péché» à la «repentance», la phrase enfle sous l'accumulation des syntagmes qui reflète la cohorte des «corruptions» de l'homme pécheur.

26. Serm. II, p. 20.

27. Serm. II, p. 18.

28. Voir G. Sicard-Arpin, "La Bible dans les sermons protestants».

29. Serm. II, p. 28.

3o. Serm. I, p. 8. 
Leffet de chute en fin de phrase n'en est que plus brutal, et l'utilisation morale du songe de Nabuchodonosor mobilise ce faisant les ressorts émotionnels de la peur chez l'auditeur, afin de donner à celui-ci la mesure de ses forfaits. En revanche, lorsqu'il s'agit de dresser un portrait général du pécheur, c'est dans les psaumes que Vignier trouve son modèle d'écriture, non pas qu'il revendique pour lui-même le statut prophétique de Nathan mettant en garde David:

Nathan ne t'est pas envoyé; mais David luy-mesme t'est proposé, afin que sa blessure soit ta guerison, \& son naufrage ton port de salut. L'ois-tu crier? crie avec luy: L'ois-tu lamenter? Join tes larmes aux siennes. Lois-tu prier? Prie avec luy ${ }^{31}$.

Au contraire, Vignier semble chercher à s'effacer derrière la voix des prophètes de l'Ancien Testament et à s'inclure lui-même dans la foule des pécheurs anonymes que ces voix doivent conduire à l'humilité et à la repentance:

Il nous en prend à tous comme à David. Ta perdition vient de toy, ô Israël, mais ton salut est du Seigneur Dieu, \& de l'Agneau qui est assis avec luy sur le trône ${ }^{32}$.

Et ce sont aussi bien les mots de David ${ }^{33}$ dans les psaumes que son expérience propre qui servent au prédicateur de trame pour décrire toute l'horreur du péché. Ainsi trouve-t-on par exemple, dans le deuxième

3I. Serm. III, p. 33.

32. Serm. I, p. 12. Vignier reprend ici une citation du prophète Osée qu'il a exposée dans son épître dédicatoire, et qui peut donc apparaître sans être référencée: "Ta perdition est de toy, ô Israël ; mais en moy est ce qui te peut aider, dit l'Eternel en Osée» [Osée 13.9, en marge].

33. Les citations de psaumes à l'intérieur des sermons sont nombreuses, sans être évidemment les seules références scripturaires. Il faudrait en outre ajouter à ces citations explicites, dont les références apparaissent dans le corps du texte ou dans ses marges, tous les cas de réminiscences ou de citations implicites, où les mots des psaumes affleurent à la surface du texte. Au huitième sermon par exemple, lorsque Vignier écrit: "Nous sommes en ce monde en une valée de mort, il nous faut passer à travers de continuelles afflictions, le temps n'est point encor venu que nous montions vers nôtre Pere, $\&$ qu'il nous fasse jouïr de sa glorieuse presence en laquelle est le plein rassasiement de joye [en marge: Pseau. 16.11]", l'environnement citationnel du psaume 16 fait reconnaître d'autant plus aisément une allusion en début de phrase à la "vallée d'ombre de mort" du psaume 23. Nous avons choisi de nous intéresser ici davantage aux images quaux citations psalmiques en tant que telles, mais il est évident qu'une étude des usages de 
sermon, cette définition paradoxale de la misère opposant prospérité apparente et pauvreté intérieure, que le psaume 32 présente sous forme de maxime:

Le comble de misere c'est le péché. Quand tu serois plus pauvre que Lazare, plus plein d'ulceres que luy, si tu es assuré de la remission de tes pechez, tu es hors de misere. O que bien-heureux est l'homme auquel Dieu n'impute point son péché, dit David Ps. 32. Comme au contraire quand tu serois vestu plus somptueusement $\&$ traité plus magnifiquement que luy, quand tu serois honoré \& adoré de tout le monde, si tu demeures en ton péché, tu n’es qu'une miserable creature ${ }^{34}$.

Dans le sermon suivant, Vignier reprend cette même idée, mais en l'illustrant directement à partir de l'exemple de David:

L'acte du péché de David estoit passé, mais la soüillure en demeuroit. Le plaisir s'en estoit allé, mais sa conscience ne cessoit pas de l'accuser, de le condamner, de le tourmenter, \& toute la gloire de son Royaume, toute la pompe de sa Cour, tous les delices de Canaan n'y pouvoient pas donner remede ${ }^{35}$.

Cette "soüillure» interne qui ronge l'être insidieusement et qui est invisible à tout autre qu'au pécheur lui-même, instaure tout un réseau de métaphores - héritées en partie de saint Augustin - autour du registre médical de la "gangrène" et de l'«ulcère" ${ }^{36}$, ainsi qu'une constellation de représentations sensorielles du péché: registre olfactif

la citation permettrait de renforcer la prégnance de la rhétorique des psaumes chez Vignier.

34. Serm. II, p. 24.

35. Serm. III, p. 34-35.

36. Voir par exemple Serm. III, p. 42: «David fut blessé par l'œil, \& cette blessure tourna en une gangrene d'adultere, \& de meurtre bien difficile à guerir. Il n'y a soüillure, il n'y a ulcere, il n'y a chancre, il n'y a lepre si abominable aux yeux des hommes, comme est le peché devant Dieu»; Serm. IV, p. 62: "Ainsi faut-il que le corrosif de la douleur Chrêtienne estant appliqué une fois aux playes pourries de nos péchez, demeure avec nous, jusqu'à ce que nous soyons du tout gueris»; Serm. VI, p. 86: «depuis le sommet de nôtre chef, jusques à nos affections, qui sont comme la plante de nos pieds, il n'y a rien d'entier en nos ames mêmes, il n'y a que playes, il n'y a qu'ulceres ». 
de la "puanteur» ${ }^{37}$, registre visuel de la «tache» indélébile ${ }^{38}$, registre gustatif de l'amertume, qui va de l'aigreur au poison ${ }^{39}$. Or sur ce point précisément, le texte rejoint l'une des images privilégiées du livre des psaumes: car, comme dans les psaumes de déploration qui dénoncent la méchanceté des "ennemis» ${ }^{40}$, vinaigre et "fiel» disent ici l'intensité du malheur que le péché entraîne:

Le péché du commencement semble savoureux au palais, mais il se trouve enfin que c'est un levain qui enaigrit tout, $\&$ rend les choses les plus douces ameres ${ }^{41}$.

C'est là ce fiel d'amertume qui a imbu, ce levain qui a enaigri, cette lepre qui a infecté non seulement toutes les parties de nos corps, mais aussi toutes celles de nos ames ${ }^{42}$.

37. Serm. II, p. 27: «le péché est chose puante, abominable $\&$ insupportable devant la face de Dieu»; Serm. III, p. 43: "le péché a infecté l'air, les champs, les eaux, les plantes, les planettes, \& couvert d'une hideuse tache toute la surface de la Nature»; Serm. VI, p. 83: «Ha mon Dieu! ce n'est pas d'aujourd'huy que je suis puant $\&$ abominable en moy-même devant tes yeux»; Serm. VIII, p. 119: "comme les mauvaises odeurs chassent les pigeons du colombier, aussi la puanteur de pechez éloigne de nous l'Esprit de grace». Voir aussi la Paraphrase sur ce pseaume que Vignier produit à la fin du recueil, après le dernier sermon: "Detourne, mon Dieu! detourne ta face de mes pechez, de peur que la puanteur d'iceux montant en tes narines, n'enflâme ton couroux pour me punir, \& pour me damner» (verset 11, p. 354).

38. Serm. X, p. 157: "chaque péché soüille le cœur, \& laisse une nouvelle tache en la conscience»; Paraphrase sur ce pseaume, p. 354, verset 12: «Mais quoy? Le More changera-il sa peau, \& le Leopard ses taches? [...] C'est l'eau de ton Esprit seule qui peut oster toutes les taches de ma noirçeur plus qu'Ethiopienne».

39. Serm. II, p. 28 : «le péché est un venin du serpent qui nous rend à bon droit abominable devant celuy qui est tout saint, \& qui a les yeux trop nets pour pouvoir voir le mal. Habac. 2.»; Serm. III, p. 42: "Le péché n'est pas une tache qui demeure au cuir seulement, c'est un poison qui passe jusqu'au cœur».

40. Voir par exemple psaume 64: «en lieu de fleches et de dards,/ paroles aigrement jettées/ ont atteintées»; ou psaume 69: «ils m’ont donné du fiel en mon repas,/ et de vinaigre ont ma soif abruvée».

4I. Serm. III, p. 35. Voir aussi Serm. V, p. 80: «Celuy qui garde en son cœur les plaisirs du péché ne sçauroit gouter des joyes de Dieu. Pourrois-tu mettre du miel en un vaisseau plein de vinaigre? Répens premierement ce que tu as, \& puis tu recevras ce que tu n'as pas".

42. Serm. VI, p. 85. 
Et comme dans les psaumes, à la déploration désespérée répond l'assurance du salut, au "gouffre d'épouvantement, de trouble \& de confusion» succède le «trone de grace» ${ }^{43}$ :

Il n'y a breuvage d'affliction, si amer soit-il, qui ne soit adoucy par cette joye, \& par cette liesse du salut de Dieu ${ }^{44}$.

Vignier joue des effets de la solidarité des contraires que l'on rencontre dans de nombreux psaumes: à un premier mouvement de plainte et de doléances sur l'inévitable corruption de la nature humaine répond un second mouvement qui inverse la dynamique, par la promesse que la rédemption sera accordée au pécheur repentant. Car seule la pénitence permet d'enrayer ce processus de dépérissement de tout l'être que le péché inaugure, cet engrenage conçu par le Diable qui attire l'être humain, le séduit et l'entraîne à sa perte. Au commencement, il y a Satan bien souvent, «ce subtil empoisonneur", qui «sucre» à tel point «le breuvage " qu'il le fait «aisément» avaler à n'importe qui : «mais pour avoir perdu son goût, il n'a pas perdu son venin: Et le poison est toûjours poison, quand bien il seroit présenté en une coupe d'or " ${ }^{45}$. La satisfaction du péché est éphémère, et son intensité n'est rien en comparaison de la profondeur des souffrances qui suivent l'éveil des remords de la conscience. Ou pour le dire autrement, le péché n'est que le premier acte d'une longue et douloureuse agonie, qui demande à être mise en perspective pour être saisie dans toute son étendue:

Le péché, avant qu'il [David] le commît estoit devant luy, luy souriant, l'attrayant, l'allechant comme une paillarde, qui invite ses amoureux, tellement qu'il cherche l'occasion pour le commettre. Apres l'avoir commis, il est encor devant luy, mais avec un regard triste $\&$ hideux, $\&$ pourtant il cherche à le cacher pour ne le point voir: Et maintenant sa conscience estant éveillée, le peché se tient devant luy tout de front, qui l'accuse, qui le tourmente, qui le fait crier \& lamenter. [...] Avant l'action c'est un ennemy qui nous flatte pour nous tuer sous ombre d'amitié. En l'action, c'est un doux poison, mais mortel, que le sens avale avec plaisir. Apres l'action, c'est un Scorpion qui picque de sa

43. Serm. XIX, p. 310.

44. Serm. XII, p. 193 sq.

45. Serm. III, p. 35. 
queuë, \& ne laisse que des horreurs, des terreurs, des angoisses au cœur, en la conscience, $\&$ en l'esprit ${ }^{46}$.

La dogmatique de Vignier n'a rien d'original en la matière: nos péchés actuels ne sont que des avatars redoublés du péché originel, des "fruicts" de cette corruption première, "generale» et "universelle " ${ }^{47}$, qui rendent nos "transgressions actuelles" doublement condamnables ${ }^{48}$. Il faut donc «travailler de bonne heure à dompter \& corriger cette corruption de la nature " ${ }^{49}$, mieux encore, il faut faire mourir "nôtre nature corrompuë, que l'Ecriture Sainte appelle «Vieil homme» pour laisser place au «Nouvel homme», selon la terminologie de l'épître aux Ephésiens ${ }^{50}$ :

En un mot, c'est un changement $\&$ conversion de l'homme tout entier, à sçavoir du péché à la sanctification, des tenebres à la lumiere, de la mort à la vie, de la puissance de Satan à Dieu ${ }^{51}$.

En revanche, c'est dans la recherche constante d'un registre visuel qui doit beaucoup à la tradition rhétorique de l'enargeia ${ }^{52}$ que la prose de Vignier révèle toute son originalité, pour faire comprendre les "tristes $\&$ mal-heureux effets du péché " ${ }^{53}$, en particulier dans le choix de comparaisons animalières qui ne sont pas bibliques, ou dont le lien biblique s'est perdu dans la présentation qu'en donne Vignier. Le scorpion que nous avons évoqué en est un bon exemple ${ }^{54}$, mais il est loin

46. Serm. IV, p. 59.

47. Expressions que l'on rencontre très fréquemment chez Vignier; voir par exemple Serm. VI, p. 83, 85 et 98 .

48. Voir par exemple Serm. VI, p. 85: "Car tout ainsi que celuy qui a commis un meurtre estant ivre, est doublement punissable, \& à cause de son ivrognerie, \& à cause du crime qu'il a commis en icelle. De même en est-il icy: Nous sommes dignes de double condamnation: premierement à cause de cette corruption de nôtre nature, puis à cause des fruicts de cette corruption en nos transgressions actuelles".

49. Serm. VI, p. 90.

50. Serm. X, p. 166. Voir aussi Serm. VI, p. 98.

5I. Serm. X, p. 166.

52. Voir P. Galand-Hallyn, Les Yeux de l'éloquence, p. 99-121.

53. Serm. XV, p. 247.

54. Le scorpion appartient au bestiaire de l'Apocalypse (voir Serm. I, p. 4: «Ainsi toy, ô homme! qui que tu sois, tu trouveras toûjours que les delices \& plaisirs perissables de peché finissent en douleurs. Ce sont comme ces sauterelles de l'abyme, qui ont comme des faces d'hommes, des cheveux comme cheveux de femmes, \& une queuë de scorpion 
d'être isolé: "pigeon ${ }^{55}$, "pelican ${ }^{56}$, «seiche» ${ }^{57}$, «milan ${ }^{58}$, «coq ${ }^{59}$ et "éléphant» ${ }^{60}$ constituent autant de figures incongrues de cet étonnant bestiaire ${ }^{61}$. Mais on est frappé surtout de la récurrence des images aquatiques sous la plume du prédicateur: comme celui des psaumes, l'imaginaire de Vignier se révèle instable et mouvant, baigné d'eaux et de

qui picque à la mort", Apoc. [9], 7. 8. 9), mais on voit bien dans l'exemple cité plus haut (Serm. IV, p. 59) que Vignier tend à détacher l'image de son contexte biblique.

55. De même que pour le scorpion, le pigeon, qui appartient quant à lui au bestiaire des psaumes (voir Psaume 68, dans la traduction de Bèze: «Vous reluirez comme feroit/ l'aile d'un pigeon qui seroit/ de fin argent brunie»), n'a plus rien de sa référence biblique dans l'image qu'en donne Vignier ("comme les mauvaises odeurs chassent les pigeons du colombier, aussi la puanteur de pechez éloigne de nous l'Esprit de grace», Serm. VIII, p. 119).

56. Serm. VII, p. 106: "Il y a un sang qui nourrit: comme le Pelican nourrit ses petits de son sang: ainsi Christ nourrit nos ames à salut par son sang».

57. Serm. IX, p. 139: "Comme la seiche épend de sa bouche une liqueur noire comme ancre dans la mer, pensant se cacher sous icelle, \& par cela même elle fait au contraire tant mieux remarquer au Pescheur le lieu où il la trouvera: Aussi celuy qui veut cacher son péché par un autre péché, le découvre tant plus à Dieu, \& plus le pecheur s'efforce de cacher subtilemnt son péché, \& plus Dieu le regarde d'un œil plus exact $\&$ rigoureux".

58. Serm. VI, p. 93: «Encor qu'un Milan vole bien haut en l'air si ne tourne-il point ses yeux vers le Ciel, mais les tient toûjours bandez sur la proye qu'il a découverte en bas, afin de fondre dessus plus impetueusement. Aussi quelque vol que semblent prendre les hypocrites par la hautesse de leurs contemplations \& par leur sainteté affectée, ils n'ont rien moins que leurs cœurs élevez à Dieu, mais ne cherchent que la gloire du monde, ou quelque autre proye en la terre»; Serm. IX, p. 141 : «[Dieu] ne detourne point si-tôt sa face de dessus nous, que nôtre ennemi spirituel ne soit plus prêt de se jetter sur nous que le Milan sur les poussins, lorsque leur Mere n'y prend pas garde».

59. Serm. XIII, p. 208: "Comme le coq s'éveille luy-même \& secouë la poudre de ses ailes, \& puis se met à chanter \& réveiller les autres; aussi les Ministres de la Parole de Dieu doivent se réveiller eux-mêmes, secoüer la poudre de leur negligence, \& se nettoyer des ordures des vices pour exciter les autres à faire le semblable».

6o. Serm. XIX, p. 310: "Comme l'Elephant se sert de sa trompe, \& pour armes \& pour tous offices; s'il passe les rivieres, \& qu'il se sente enfoncer par la pesante masse de son corps, il leve en haut sa trompe, \& respire l'air avec icelle: Il en est de même de la priere au fidele, elle luy sert pour tous offices, $\&$ pour toutes armes contre ses ennemis, ce luy est un aide en ses necessitez, un sacrifice envers Dieu, \& un glaive contre Satan ».

6I. Indépendamment du bestiaire, d'autres images concrètes émaillent la prédication de Vignier, mais elles sont souvent plus convenues, celle de l'horloge par exemple, intéressante par la régularité et la perfection de ses mouvements quand tout est en bon état de marche, mais où la moindre faille provoque un dérèglement général du mécanisme d'ensemble (voir Serm. II, p. 22, et Serm. XV, p. 246). 
courants contraires, hanté par le flux et le reflux de flots aussi puissants les uns que les autres, qui se donnent ensemble dans leur réversibilité symbolique ${ }^{62}$.

\section{Sources et ruisseaux}

Tout passe, tout s'écoule chez Vignier et cet imaginaire du mouvement est inscrit au seuil même de son ouvrage, comme si l'épître dédicatoire cherchait à conjurer la volubilité et l'inanité des paroles humaines par l'acte de publication du sermon:

La voix bien souvent se perd parmy un nombre d'Auditeurs, \& ce que tu as entendu à la haste, s'écoule comme la liqueur qui passe par un entonnoir.

Il faut au contraire, explique Vignier, faire de notre mémoire une "Arche pour garder les choses saintes", au lieu d'un "sas qui laisse passer la fleur, $\&$ ne retient que le son ${ }^{63}$. Seule une âme solidement instruite de la crainte des "Jugemens de Dieu " pourra faire barrage au «flot» de corruption qui l'entraîne inévitablement à sa perte. Et c'est en effet par des images grandioses sans commencement ni fin (fontaine jaillissante, mer immense, torrent impétueux), et confinant au sublime, que Vignier cherche à décrire l'irrépressible force du péché en l'homme:

Sa misere comme la mer, s'enfle par dessus les plus hautes montagnes, $\&$ atteint jusques aux parties plus reculées de cette terre, c'est à dire de l'homme, qui de soy-même n'est que poudre pêtrie \& dêtrempée en terre avec des larmes ${ }^{64}$.

Et si l'on veut remonter "premierement jusques à la source $\&$ premier origine » ${ }^{65}$ de cette misère, c'est-à-dire au péché lui-même, on trouve alors une intarissable "fontaine», "de laquelle découlent tous les ruisseaux de corruption qui sont en nous", comme une "matrice" nourricière "qui

62. Voir I. Kirschleger, "'Mon âme a soif de toi" ".

63. "Epître dédicatoire», [n. p.].

64. Serm. VI, p. 82.

65. Ibid., p. 83. 
produit tous les monstres de pechez actuels que nous commettons " ${ }^{66}$; chez certains, ces "ruisseaux" sont de véritables torrents, et, disent-ils, "la corruption de la nature nous entraîne avec violence \& impétuosité comme un rapide torrent $"{ }^{67}$. Vignier met ici à profit, en exploitant la nature mortifère des eaux psalmiques, ces images de liquidité et de fluidité qui courent au long des psaumes pour désigner la splendeur et la crainte qu'inspire la création divine. Et de même que dans les psaumes, l'orant définit ses états d'âme et ses angoisses à l'aide de métaphores fluides et aquatiques ${ }^{68}$ :

Nôtre entendement est un ocean d'ignorance, incapable des choses spirituelles, mais assez vif \& aigu au mal. [...] Nôtre mémoire est labile \& fluide comme eau, pour recevoir \& retenir les bonnes impressions, mais est comme un marbre pour retenir ce qui est mauvais. Nôtre volonté est ployable \& obéissante au Diable, c'est comme de la cire entre ses mains. Mais elle est plus dure que fer entre les mains de Dieu ${ }^{69}$.

Vignier reprend également aux psaumes l'image de la cire, en lien avec la labilité et l'inconsistance qui caractérisent selon lui la faiblesse des facultés intellectuelles ${ }^{70}$. Ainsi, «l'impureté \& corruption» de notre "rude \& sauvage nature» produit de tels débordements qu'il faudra bien que Dieu manifeste sa justice, et Vignier va donc faire valoir à maintes reprises

66. Ibid., p. 85 sq.

67. Ibid., p. 84.

68. Le champ métaphorique des psaumes est large et va de l'engloutissement par noyade ou submersion, à la liquéfaction par dispersion ou écoulement, jusqu'à l'évaporation ou la sécheresse intense. Voir Psaumes 22 («ma vertu comme eau s'escoule toute:/ n'ay os qui n'ay la jointure dissoute»), 39 ("je sen fondre mon cœur,/ sentant de ta main la rigueur»), ou 69 ("les eaux m’ont saisi jusques à l'ame,/ et au bourbier tresprofond et infame,/ sans fond ne rive enfondré je me voy./ Ainsi plongé, l'eau m'emporte»). Voir également ci-dessous note 72 .

69. Serm. VI, p. 86.

70. Dans les psaumes en effet, l'angoisse de la liquéfaction ou de la dissolution que ressent l'orant est traduite par l'image de la cire qui s'écoule et qui fond (Psaume 22, «et comme cire en moy fond goute à goute/ mon cœur fasché»), image célèbre dans les psaumes en raison de son autre emploi métaphorique dans le psaume dit "des batailles" pour désigner la dispersion des ennemis et la victoire de Dieu (Psaume 68, "comme la cire aupres du feu,/ ainsi des meschans devant Dieu,/ la force est consumée»). 
cette hantise du Jugement dernier; dès l'épître liminaire ${ }^{71}$, l'auteur semble habité par le souvenir du Déluge, et ses exhortations s'emploient à conjurer le retour d'un tel cataclysme. A ce déluge menaçant, qui est l'un des leitmotive du psautier dans son ensemble ${ }^{72}$, Vignier tente donc de substituer par petites touches de "gracieuses pluyes d'instruction" qui visent à éloigner "la malédiction de Dieu " ${ }^{73}$, et à redonner fertilité et fécondité à l'âme du fidèle ${ }^{74}$. Ainsi s'explique dans le texte l'insistance sur l'image de l'aspersion:

[...] comme nous avons plusieurs taches, aussi avons nous besoin de plusieurs goutes de ce sang. Une goute pour l'avarice, une goute pour l'intempérance, une goute pour la haine contre nos freres; des goutes, mais des flots pour effacer tant d'execrables blasphemes, qui se vomissent tous les jours contre son S. Nom ${ }^{75}$.

Et si cette image prend certes appui sur une paraphrase du psaume 51 :

Toy seul, ô mon Dieu! me peut purger \& nettoyer de péché. Arrousemoy donc, asperge-moy je te supplie avec la vraye hyssope, le sang de l'Agneau sans macule, qui en la plenitude des temps doit estre immolez pour nos pechez, \& pour ceux de tout le monde ${ }^{76}$.

7I. «Si le monde ancien n'eust esté plongé en une impie \& profane sécurité, il n'eust pas esté submergé par un deluge general ", Epître dédicatoire, [n. p.]; "Car quel a esté le déluge ancien, qu'une hydropisie des cieux, qui noya le premier monde à cause du péché?", Serm. III, p. 43.

72. Voir Psaumes 42 ("Tous les grands flots de ton onde/ par dessus moy ont passé»), 69 ("Sauve-moy car les eaux m’ont saisi jusques à l'âme»), 88 ("deluges terribles/ me tiennent tous les jours pressé»), 144 ("Tends-moy d'en haut ta main qui me delivre,/ de ces grand's eaux rends-moy sain, et delivre»).

73. Serm. VI, p. 100.

74. Voir Serm. VI, p. 99-100, et Serm. VII, p. 101 : «Fay profit de la connaissance de la vérité que tu as receuë, de peur qu'apres maintes gracieuses pluyes d'instructions, ton ame demeurant sterile, \& ta vie sans fruit, tu ne sois exposé à la malédiction de Dieu "; "C'est le péché qui nous ferme le ciel, qui nous rend la terre maudite, qui assujettit l'homme à toutes sortes de miseres, au dedans, au dehors, en cette vie, $\&$ en l'autre».

75. Serm. VII, p. 116. Le goutte-à-goutte est également une image psalmique que l'on trouve au psaume 6 ("en eau goutte à goutte/ s'en va ma couche toute,/ par si fort larmoyer»). Voir aussi Psaume 22 ("comme cire en moy fond goute à goute/ mon cœur fasché»).

76. Serm. VII, p. 106. 
elle fait plus largement écho à d'autres psaumes qui disent la nécessité d'une pluie bienfaitrice et salvatrice sur une terre aride et asséchée, comme peut l'être l'âme du fidèle, au psaume 65 par exemple:

Si la terre est de soy tarie,

tu la viens visiter,

et les grans thresors de ta pluie

sur elle degouter.

[...] Ses rayons ennivres et trempes,

Ses sillons applanis:

tu l'amollis et la detrempes,

et son germe benis.

Et c'est bien lorsque l'âme est "vuide du saint Esprit» que la menace est la plus grande d'être "submergée au milieu des flots de ses propres passions et affections " ${ }^{77}$. Mais le royaume céleste a pourtant ses propres ruisseaux et sa propre source qui suffiraient à remplir en abondance l'âme du fidèle, et c'est sur cette même solidarité des contraires que joue le texte dans son ensemble: aux eaux de Mériba ou "eaux de querelle» dans les psaumes ${ }^{78}$, devenues "eaux de tentations ${ }^{79}$ et $"$ ruisseaux de corruption" répondent ainsi des "ruisseaux de compassion» ${ }^{80}$ qui découlent des «fontaines d'eaux vives» où "puiser des eaux de salut en joye» ${ }^{81}$ :

Il n'est besoin que les pecheurs viennent à cette fontaine comme on venoit au lavoir de Bethesda un à un; quand vous y viendriez en aussi grand nombre qu'il y a d'estoiles aux Cieux, ou de grains de sablon au rivage de la mer, vous y trouverez remede à toutes vos miseres, $\&$ une medecine pour toutes vos playes. Il y a icy un Jubilé continuel. Il y a icy une fontaine toûjours ouverte pour le peché \&o pour la soüillure ${ }^{82}$.

On voit ici une fois encore à quel point l'imaginaire des psaumes, pardelà les seuls mots du psaume 51, a imprégné profondément la prose

77. Serm. XII, p. 199.

78. Voir par exemple les psaumes 73, 81, 95 et 106.

79. Serm. XIX, p. 311.

80. Voir Serm. II, p. 31: "Et que les ruisseaux de tes compassions qui ont si abondamment découlé sur tant d'autres, ne soient point seichez pour moy».

8I. Serm. VI, p. 86.

82. Serm. III, p. 34. 
de Vignier: le prédicateur se souvient combien les psaumes répètent que les «ruisseaux» de Dieu «regorgent» d'eau (psaume 65), que son "palais vousté" est "lambrissé d'eaux" (psaume 104), et que par-là même, "source de vie en [lui] gist» (psaume 36). On ne s'étonne donc guère de constater que Vignier a recours de même à une métaphore végétale et organique pour définir les véritables qualités du chrétien:

Ainsi les vrais Chrêtiens doivent estre saints au dehors: [...] Mais beaucoup plus doivent-ils estre saints au dedans: Il faut que le Seigneur reside au plus secret de leur ame, \& que le temoignage du Seigneur, c'est à dire sa parole, habite plantureusement en eux ${ }^{83}$.

L'adverbe "plantureusement" éveille ici le souvenir des psaumes qui définissent les justes comme les "heureuses plantes de la maison de Dieu", florissantes "au beau milieu des parvis" et "verdoyant chacun an» (psaume 92), selon cette promesse que le psaume 1 énonçait déjà avec une ferme assurance: "qui au conseil des malins n'a esté/ [...] semblera un arbre grand \& beau/ planté le long d'un clair courant ruisseau/ et qui son fruict en sa saison apporte».

Avec subtilité et cohérence, Vignier nous offre ainsi l'exemple abouti d'une prédication qui cherche à donner une résonance imaginative concrète à un enseignement dogmatique des plus traditionnels, à savoir la corruption première et intrinsèque de la nature humaine depuis le péché d'Adam, d'où le prédicateur tire la nécessité d'une prompte et permanente repentance. Pour frapper l'esprit de l'auditeur (et du lecteur) par des images fortes, surprenantes parfois, saisissantes souvent, sans rompre toutefois l'harmonie d'ensemble du recueil, Vignier pratique une écriture en système, où les sermons se répondent et se complètent par les effets de miroir qui les portent, et qui trouvent dans les psaumes l'un des moyens privilégiés de ce jeu de correspondances. La Pratique de Repentance témoigne ainsi de l'importance que revêtait pour les réformés d'alors le psautier dans son intégralité, par-delà le choix d'un psaume précis comme support de la prédication: l'imaginaire labile du psautier innerve d'un bout à l'autre les méditations de Vignier, dans la fidélité aux images et aux symboles psalmiques. Mais c'est là une constante de la prédication réformée d'une façon générale, et l'usage du livre des psaumes par

83. Serm. X, p. 163-164 (nous soulignons). 
Vignier n’a rien de spécifique à sa génération : la prédication de Vignier illustre avec force cette pratique de la «langue de Canaan» si chère au cœur réformé qui puise dans les Ecritures la matière de sa propre pensée et de sa profession de foi. Seulement, il est vrai que la méditation de Vignier sur l'œuvre du temps en matière de corruption spirituelle, la présence appuyée d'un bestiaire original, de même que le travail du texte sur les images métamorphiques correspondaient bien à cet imaginaire d'époque que l'on qualifie parfois de «baroque». On pourrait de même rattacher à cette inspiration toutes les images qui traitent de l'apparence trompeuse ou cachée des choses, et qui reviennent assez fréquemment sous la plume du pasteur, qu'il s'agisse des "sépulcres blanchis» ${ }^{84}$, ou d'images plus prosaïques opposant une extériorité lisse et sans heurt à une intériorité gâtée et disgracieuse ${ }^{85}$. Ces quelques accointances "baroques" suffiraient-elles à expliquer le succès indéniable de l'ouvrage? Nul doute en tout cas que l'œuvre de Vignier s'inscrit incontestablement dans cette célébration rhétorique de l'excellence des images et de l'utilité pédagogique des psaumes qui parcourt tout le XVII ${ }^{\mathrm{e}}$ siècle, au cœur de cette certitude que lorsque «l'esprit de l'homme soûpire», toujours "l’Esprit de Dieu l'inspire» ${ }^{86}$.

\author{
Inès KirSCHLEGER \\ Université de Toulon
}

84. Voir Serm. XIII, p. 216: «Mais le pecheur non repentant n'a de beauté qu'en l'apparence externe, comme les sepulcres blanchis, apparoissent beaux par dehors, mais par dedans ils sont pleins d'ossemens de morts, \& de toute ordure [Matthieu 13.27]. Bref tel est le desordre, telle la confusion de ce miserable, qu'il fait horreur à soy-même, s'il se regarde au miroir de la Loy de Dieu, $\&$ ne peut voir que les enfers ouverts pour l'engloutir".

85. Voir Serm. VI, p. 98: «Ne soyons point comme ces boëtes qui sont quelquefois és boutiques des Apoticaires, bien dorées $\&$ peinturées par dehors avec de beaux écriteaux \& dedans il n'y a rien, ou n'y ont que quelque vieille drogue éventée, ou quelque poison»; Serm. XVII, p. 283: "Et comme quand une noix, ou une amande est froissée $\&$ cassée, ce qui estoit caché dans la coque paroit alors, \& on voit à découvert ce qui estoit dedans. Ainsi en l'ame vrayement humiliée $\&$ repentante, l'esprit froissé met hors ces pechez qui estoient cachez \& couverts, \& n'a point honte de les decouvrir, \& aux yeux de Dieu, \& aux yeux des hommes mêmes, comme vous voyez ici en l'exemple de David".

86. Serm. XVII, p. 289. 


\section{BIBLIOGRAPHIE}

\section{Textes}

Aubigné, Agrippa d', Meditation sur le Pseaume LI, in Cuvres complètes, tome I, Petites CEuvres meslees [1630], éd. par Véronique Ferrer, Paris, Honoré Champion, 2004, p. 247-273.

Bèze, Théodore de, Meditation sur le LI Psalme, in Chrestiennes Meditations sur huict Pseaumes du prophète David [1581], éd. par Mario Richter, Genève, Droz, 1964, p. 71-78.

Daillé, Jean, Mélange de sermons. Prononcés par Jean Daillé à Charenton pres de Paris, en divers temps, \& sur differens sujets, Amsterdam, Jean de Ravesteyn, 1658.

Du Bosc, Pierre, Sermons sur divers textes de l'Ecriture sainte convenables au temps, Rotterdam, Reinier Leers, 1701.

Duplessis-Mornay, Philippe, Meditation sur le Pseaume LI [1595], in Méditations sur les psaumes, éd. par Pascale Blum-Cuny, Paris, Honoré Champion, 2004, p. 119-176.

Marot, Clément et Bèze, Théodore de, Les Psaumes en vers français avec leurs mélodies, fac-similé de l'édition genevoise de Michel Blanchier, [1562], éd. par Pierre Pidoux, Genève, Droz, 1986.

Morus, Alexandre, Sermons choisis de Monsieur Morus sur divers textes de l'Ecriture sainte, Genève, Cramer \& Perachon, 1694.

Mussard, Pierre, Sermons sur divers textes de la Saincte Escriture, Genève, Pierre Chouët, 1673.

Turrettin, François, Sermons sur divers passages de l'Escriture Sainte, Genève, Samuel de Tournes, 1676.

Vignier, Nicolas, Theatre de l'Antechrist, auquel est répondu au cardinal Bellarmin, Paris, [s. n.], 1610.

—, Le Pescheur d'hommes, ou Du devoir \& des qualitez des Ministres de la Parole de Dieu. Avec le formulaire de la Reception \& Confirmation 
des Pasteurs, selon la Discipline des Eglises Reformées de ce Royaume, Blois, Veuve Gaucher Collas, 1632.

-, La Pratique de Repentance, ou Sermons sur le Pseaume 51, avec une paraphrase d'iceluy. Par Nicolas Vignier. ITEM La Recherche du Coeur, ou cinq Sermons sur le 10 verset du 17. chapitre de Jeremie. Le Bon Centenier, ou cinq sermons sur sept versets du chap. 8 de $S$. Mathieu. Par Nicolas Vignier son Fils, tous deux Ministres du S. Evangile en l'Eglise Reformée de Bloys. Se vend à Charenton, Par Estienne Lucas, [s. 1.], [s. n.], 1670.

Viguier, Jérémie, L'ouverture des levres ou Sermon sur le Pseaume 51. vers. 17. Par Jeremie Viguier Ministre de la Parole de Dieu en l'Eglise Réformée de Nerac, Genève, G. Lafargue Imprimeur \& Marchand libraire, 1645.

\section{Travaux}

Ferrer, Véronique, «Variations autour du Psaume 51: les méditations de Bèze, d'Aubigné et de Duplessis-Mornay", Bulletin de la Société de l'Histoire du Protestantisme français, 149/IV (2003), p. 705-717.

Galand-Hallyn, Perrine, Les yeux de l'éloquence. Poétiques humanistes de l'évidence, Orléans, Paradigme, 1995.

HaAg, Eugène et Emile, La France Protestante ou vie des protestants français qui se sont fait un nom dans l'Histoire [1846-1859, 10 vol.], Paris-Genève, Joël Cherbuliez, 1859.

Kirschleger, Inès, "'Mon âme a soif de toi'. L'eau et la soif dans les Psaumes, d'après la version poétique de Clément Marot et Théodore de Bèze», Foi \& Vie, Cahier Biblique, 45 (2006), p.9-26.

Sicard-Arpin, Ghislaine, "La Bible dans les sermons protestants: le fondement d'une identité, l'arme contre l'adversaire catholique», in Bible et littérature, éd. par Olivier Millet, Paris, Honoré Champion, 2003, p. 111-119.

—, "Le sermon protestant: un enracinement dans la parole de Dieu», Le temps des beaux sermons, éd. par Jean-Pierre Landry, Genève, Droz, 2006, p. 51-59. 